\title{
An Electron Model Consistent with Electron-Positron Pair Production from High Energy Photons
}

\author{
Donald Bowen' ${ }^{1}$, Robert V. Mulkern ${ }^{2}$ \\ ${ }^{1}$ Independent Researcher, Concord, MA, USA \\ ${ }^{2}$ MRI Researcher, Brigham and Women's Hospital, Boston, MA, USA \\ Email: quark377@aol.com
}

Received 11 June 2015; accepted 24 August 2015; published 27 August 2015

Copyright (C) 2015 by authors and Scientific Research Publishing Inc.

This work is licensed under the Creative Commons Attribution International License (CC BY). http://creativecommons.org/licenses/by/4.0/

(c) (i) Open Access

\begin{abstract}
This is a rotating charge loop model of an electron which explains the electron's de Broglie base frequency to an accuracy of over 6 decimal places. The model also predicts the magnetic moment of the electron to over 6 decimal places and helps explain the transition from a purely electromagnetic photon to a fermion state of matter. The model also explains how charge and spin are conserved in the transition. Finally, this concept might be extended to explain the muon and tau higher energy states of the electron as well.
\end{abstract}

\section{Keywords}

Electron, Positron, Loop, Muon, Tau, Pair Production, Photon, Fermion, Magnetic Moment, De Broglie

\section{Introduction}

In the 19th century, Maxwell showed that light is composed of electromagnetic energy [1], a concept later refined by Einstein to explain the photonic individual wave-packet nature of its travel. In the early 20th century, de Broglie extended the concept to show that matter, like the electromagnetic photon, also has a wave nature. Light and electrons are similar, and one can convert to the other, i.e., high energy photons (>1.022 MeV) can be converted into matter in the form of electron-positron pairs (511 KeV each) when the photon encounters sufficient acceleration out of its rest frame (typically a collision with matter) [2] (see Graph 1 at the end of paper). Since de Broglie's insight, some attempts have been made to understand how an electron is formed, and although some are very intriguing, none has fully explained the transition from a purely electromagnetic photon 
state to a fermion state of matter. The following model is intended to provide a potential explanation of this transition.

The model has two parts: 1) What is the form of an electron, and 2) How does a high energy photon convert into an electron-positron pair?

\subsection{Form of the Electron}

Many attempts have been made to estimate the size and shape of an electron. At the turn of the 20th century, Lorentz presumed it to have a spherical shape and then calculated its radius to be $2.82 \times 10^{-15} \mathrm{~m}$ [3]. He assumed that its mass was due to its electrical energy and used an integral to calculate the energy required to bring the charge of an electron from infinity down to the surface of a sphere of a given radius. The radius that fulfils that energy calculation is $2.82 \times 10^{-15} \mathrm{~m}$. Lorenz however, was unaware of "Spin", which had not been discovered yet. Since his calculation was based solely on electrostatics and didn't include the energy in the magnetic spin of the electron, his calculation was necessarily incomplete. A significant problem arises if you take the Lorentz radius and calculate how fast it needs to rotate in order to produce the magnetic moment of the electron; it makes no sense because the speed of the surface would need to exceed the speed of light!

The size of an electron unfortunately cannot be measured directly (uncertainty principle), so what other means is there to estimate the diameter of the electron? We know the "Charge" of an electron to at least 7 decimal places, and we also know the bare Magnetic Moment of the electron to about the same accuracy. If we take those two extremely accurate figures and assume that the electron's charge rotates in a circle at the speed of light, and then solve for the diameter required to generate the magnetic moment of an electron, we arrive at a diameter $d=7.72318492 \times 10^{-13} \mathrm{~m}$.

Calculations for above:

Known values and definitions:

$Q_{e}$ (charge of electron) $=1.602176565(35) \times 10^{-19}$ Coulombs [4];

$\mu_{e}$ (bare magnetic moment electron $)=9.27400968(20) \times 10^{-24} \mathrm{Amp} \cdot \mathrm{m}^{2}[5]$;

$g(g$ factor correction $)=1.00115965218073[6]$;

Note: see Postulate 4) below for when and why to apply $g$ factor correction.

$C$ (speed of light $)=2.99792458 \times 10^{8} \mathrm{~m} / \mathrm{s}$;

$T$ (transit time at speed $C$ around loop of diameter $d$ ) $=\pi d / C$;

$\mu_{l}$ (magnetic moment of current around any circle) $=$ Amperes $\times \pi d^{2} / 4$.

For a charge of a single electron going in a circular loop at the speed of light:

$$
\begin{gathered}
A m p_{e}(\text { Ampere around loop })=\text { Coulomb } / \mathrm{s}=\left(Q_{e} / T\right)=Q_{e} /\left(\frac{\pi d}{C}\right) \\
\mu_{l}=A m p_{e} \times\left(\frac{\pi d^{2}}{4}\right)=Q_{e} d C / 4
\end{gathered}
$$

Solving for the " $d$ " that satisfies the known bare magnetic moment $\mu_{e}$ :

$$
\begin{aligned}
& d_{e}(\text { diameter of electron loop })=4 \mu_{e} /\left(Q_{e} C\right)=7.72318492 \times 10^{-13} \mathrm{~m} \\
& \text { Length around loop }=\pi \times 7.72318536 \times 10^{-13} \mathrm{~m}=2.426310 \times 10^{-12} \mathrm{~m}
\end{aligned}
$$

What is interesting is that the length around this loop happens to exactly match the wavelength of a photon with equivalent energy to the rest mass of an electron i.e. a $0.510998910 \mathrm{MeV}$ photon (this photon wave length is also exactly $2.426310 \times 10^{-12} \mathrm{~m}$ ).

It is as if an electron is simply a $511 \mathrm{KeV}$ photon that is curving back on itself! But this is hard to believe because a photon is neutral, i.e. it has no net charge. Williamson and van der Mark hypothesized an interesting model showing how a photon might be able to wrap back on itself [7], but their model does not show how a photon can transit to both an electron and a positron, or how it would generate the de Broglie wavelength and frequency. If, instead of assuming that the $511 \mathrm{KeV}$ photon wraps back on itself (forward to backwards), if we instead look at the original $1.022 \mathrm{MeV}$ photon as composed of a rotating charge separation, i.e. a positive and a negative charge rotating about each other, as postulated by James Maxwell [1], and then if the two charged 
components were to loop back on themselves transversally to the forward direction of the photon as shown in Figure 1 "After", then we have a simple model for the transition from a bosonic photon to a fermionic state of matter in which not only are all electrical, magnetic and spin components accounted for, both before and after the transition, but we have a model which also generates precisely the correct de Broglie wavelength and frequency of the electron and positron (see calculations in Postulate 4) below).

In formulating this model, we have been lead to propose the following postulates:

Postulate 1) A purely electromagnetic photon has "open” magnetic field lines, which oscillate, but never close on themselves, which is why it travels at the speed of light.

Postulate 2) Matter is different from a photon only in that its magnetic field lines close on themselves (allowing the energy of matter to exist at rest).

Postulate 3) The mass of matter arises from the distortion of space due to the concentration of the electromagnetic energy. Note: this is not a new idea; Lorentz and others have suggested this by equating the electromagnetic energy in an electron with its mass (and this also fits Einstein's view that the "gravity" of mass is due to a distortion of space time itself).

Postulate 4) The de Broglie matter-wave arises from the natural frequency of the electron which generates "virtual" photons of the same frequency. The photons are then reabsorbed as described by Feynman; "the electron goes along for a while and suddenly emits a photon; then (horrors!) it absorbs its own photon" [8]. The "base" frequency (at $v=0$ ) of this model exactly matches the de Broglie rest frequency (leading to an exact match with the de Broglie frequency and wavelength at any velocity when the "gamma" correction for relativity is applied):

$$
\begin{aligned}
& \text { de Broglie Frequency: } f=\frac{E}{h}=\left(\gamma m_{0} c^{2}\right) / h \text { [9] } \\
& \text { de Broglie Wavelength: } \lambda=\frac{h}{p}=h /\left(\gamma m_{0} v\right) \text { [9] }
\end{aligned}
$$$$
\text { (tied to above frequency by Planks constant } h=E / f \text { ) }
$$

$$
\text { where } \gamma=\frac{1}{\sqrt{1-v^{2} / c^{2}}} \text { is the "gamma" correction for relativity }
$$

The frequency of this model is $1 / T=C / \pi d=1.23559003 \times 10^{20} / \mathrm{s}$.

This frequency matches, to over six decimal places, the de Broglie "base" frequency (at electron velocity $=0$ )

$$
f_{\text {de Broglie }}=\frac{0.510998910 \times 10^{6} \mathrm{eV}}{4.135667517 \times 10^{-15} \mathrm{eV} \cdot \mathrm{s}}=1.2355899 \times 10^{20} / \mathrm{s}
$$

Note 1: Feynman used "virtual" photons as a way to calculate the " $g$ " factor correction to the bare electron. The virtual photon contributions that he and others calculated for $g / 2$ added a correction factor of 1.00115965246 to the bare magnetic moment (amazingly close to the experimentally measured value of $1.00115965218[8])$.
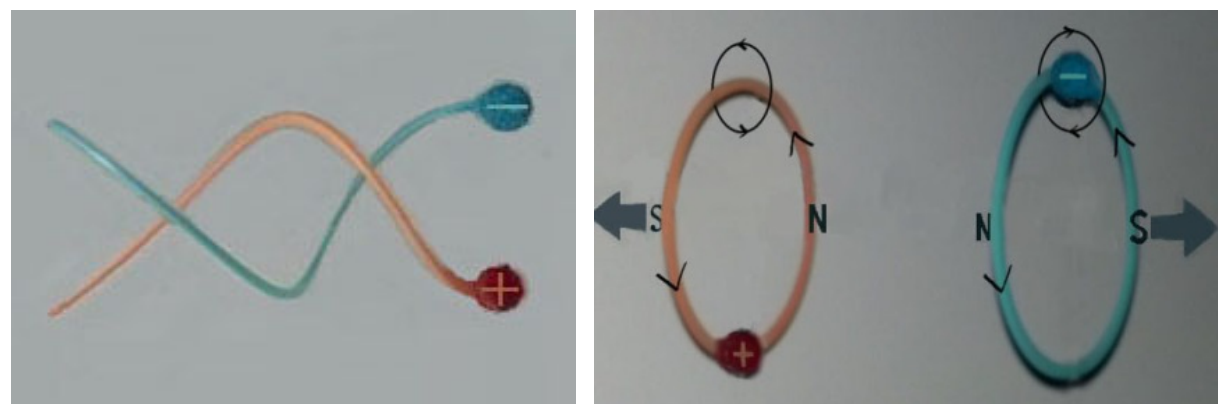

Figure 1. "Before" Photon $\lambda=12.13 \times 10^{-13} \mathrm{~m}$; “After" Loop Diam $=7.723 \times 10^{-13} \mathrm{~m}$. 
Note 2: the concept in Postulate 4) is closely related to the Broglie-Bohm "Pilot Wave Theory" [10]. In this model, the Pilot Wave is generated by the electron itself in the form of virtual photons that are emitted in the vicinity of the electron and then reabsorbed. The virtual photons here being the mechanism by which the electron "feels" and is "felt by" it's surrounding matter. In the particular case of a double slit experiment, if the electron's core loop goes through just one slit, the virtual photons surrounding the electron can go through both slits leading to an interference with itself. Note: both Einstein and de Broglie favored this type of "local" interpretation of quantum physics which was defended by Einstein in the famous Einstein-Bohr debates at the 5th Solvay conference in Brussels in 1927 [11].

\subsection{Transition from Boson to Fermion}

We have addressed the electron, but to better understand the transition from photon to matter it is important to understand the nature of the photon as well.

Figure 1 "Before" shows how Maxwell envisioned light traveling [1] as a helix of charge-separation travelling through space with the electrical and magnetic components chasing each other at the speed of light. The general form of the projection of the photon onto a plane perpendicular to its direction of travel would be an ellipse [12]. Maxwell was able to calculate the speed of light simply from the vacuum electrical permittivity constant (the capacitance like ability to separate charge in free space) combined with the vacuum magnetic permeability constant:

$$
\text { Maxwell: } \quad c^{2}=1 /\left(\varepsilon_{0} \mu_{0}\right) \quad[13]
$$

if we combine Maxwell's equation with Einstein's equation

$$
\text { Einstein: } E=\operatorname{mass} \times c^{2}
$$

we then get:

$$
\text { mass }=E \times \varepsilon_{0} \times \mu_{0}
$$

All of the terms on the right are electromagnetic, which support Postulate 3) which states that the "mass" formed is due to the distortion of space by the concentration of electromagnetic energy.

Figure 1 "Before" and Figure 1 "After" show the transition from a $1.022 \mathrm{MeV}$ photon to positive and negative loops that are closed and repelling away from each other magnetically. The positive loop is a positron and the negative loop is an electron.

Note: the wavelength in Figure 1 "Before" and the loop diameters in Figure 1 "After" are roughly in scale relative to each other, but the other dimensions such as the size, shape and distribution of the charged regions may differ from what is shown. Due to the compressive forces of the magnetic field lines inside and outside the loop, the thickness of this charged layer may be very thin and possibly sinusoidal distributed around the perimeter of the loop.

\section{Reality Testing of the Model}

Reality Test 1: One reality test of this model is that it can explain how a positron and an electron can fly away from each other. In order to overcome the extremely high electrostatic attraction between an electron and a positron at $10^{-13}$ meter distances, the magnetic alignments must be opposite at the initial formation of the electron positron pair. In this model, we hypothesize that when the photon wave frequency reaches the resonant frequency necessary for the electron and positron loops to form (loop transit frequency $1 / \mathrm{T}$ ), the newly formed loops become strong static magnets, which due to their opposed alignment at the moment of formation, will then repel apart due to the opposing magnetic field forces. They can fly apart because the magnetic repulsion will be greater than the electrostatic attraction at this length scale.

Calculation for distance at which the magnetic force $\left(F_{m}\right)$ equals the electrostatic force $\left(F_{e}\right)$

$$
F_{m}=\left(\frac{3 \mu_{0}}{4 \pi}\right)\left(\left(\operatorname{Mag}_{1} \operatorname{Mag}_{2}\right) / r^{4}\right)
$$




$$
F_{e}=\left(\frac{1}{4 \pi \varepsilon_{0}}\right)\left(Q_{e} Q_{e} / r^{2}\right)
$$

For one electron and one positron, aligned for maximum magnetic repulsion, the calculations are:

$$
\begin{aligned}
& F_{m}=\left(2.586 \times 10^{-53}\right) / r^{4} \mathrm{~N} \cdot \mathrm{m}^{4} \\
& F_{e}=\left(2.31 \times 10^{-28}\right) / r^{2} \mathrm{~N} \cdot \mathrm{m}^{2}
\end{aligned}
$$

Solving for " $r$ " at equal force we get: $3.35 \times 10^{-13}$ meter.

Hence separation can occur because the two charges are already separated by more than this distance during the formation of the loops (see diameter of photon and loops in Figure 1 "After") whereas the magnetic loops start out almost overlapping (i.e. closer together than this distance), hence forcing the loops apart.

Note: the above " $r$ " calculation can also be used to determine at what scale two electrons or two positrons can be forced together (see "quark" comments in discussion at end).

Reality Test 2: Spins add up: a spin of 1 (in photon) becomes a $1 / 2$ spin positron and a $1 / 2$ spin electron (note: all charges, all magnetic fields, and all spins add up).

Reality Test 3: A crucial reality test of this model is that it explains and exactly matches the De Broglie frequency and wavelength (see Postulate 4).

Reality Test 4: This model can also be extended to explain higher energy states of the electron such as the muon and tau.

If we extend the original concept that the loop trajectory of the charge density needs to be exactly one wave length of the original photon of equal energy (the Compton wavelength of a $0.511 \mathrm{KeV}$ photon), and then we look at the possibility of looping that same wavelength several times around a smaller diameter trajectory such that the total circumference of all the smaller loops still adds up to the Compton Wave length, then we have a relationship between energy density (mass) and magnetic moment that exactly matches the muon and tau.

This "Loop de Loop" hypothesis predicts that as the number of loops increases, the electromagnetic energy and hence mass will increase as one electron's worth of charge is concentrated into a smaller loop. Since the energy and hence mass increases in direct proportion to the inverse of the radius, and since the radius decreases in direct proportion to the number of loops $\left(\#_{\text {loops }}\right)$, we can use the following relation:

$$
\text { Mass is proportional to } \#_{\text {loops }}
$$

$$
\text { The magnetic moment is } A m p_{e} \times \text { loop area } \times \#_{\text {loops }}
$$

The area of each loop is proportional to its radius squared, which is inversely proportional to the \# of loops, which means that the net magnetic moment is proportional to

$$
\frac{\#_{\text {loops }}}{\left(\#_{\text {loops }}\right)^{2}}=1 / \#_{\text {loops }}
$$

This means that as mass increases, the magnetic moment should go down by exactly the same amount:

$$
(\text { Mass } \times \text { Magnetic moment })=\text { constant }
$$

Electron Mass $\times$ Electron Magnetic Moment

$$
\begin{aligned}
& =\left(0.510998910 \times 10^{6} \frac{\mathrm{eV}}{\mathrm{c}^{2}}\right) \times\left(9284.764 \times 10^{-27} \mathrm{Amp} \cdot \mathrm{m}^{2}\right) \\
& =4.74450 \times 10^{-18} \mathrm{Amp} \cdot \mathrm{m}^{2} \cdot \frac{\mathrm{eV}}{\mathrm{c}^{2}}
\end{aligned}
$$

Let's check the muon: 
The Muon Mass $\times$ Muon Magnetic Moment

$$
\begin{aligned}
& =\left(105.6583668 \times 10^{6} \frac{\mathrm{eV}}{\mathrm{c}^{2}}\right) \times\left(44.904478 \times 10^{-27} \mathrm{Amp} \cdot \mathrm{m}^{2}\right) \\
& =4.74453 \times 10^{-18} \mathrm{Amp} \cdot \mathrm{m}^{2} \cdot \frac{\mathrm{eV}}{\mathrm{c}^{2}}
\end{aligned}
$$

which is identical if you take into account the different " $g$ " factor corrections of each.

Notes: if we are correct in our proposal that the mass of the muon is proportional to the \# of loops (Equation (16)), then the \# of loops in a muon would be the ratio of the muon mass to the electron mass, or 206.8 loops in our time frame (slightly higher in its own time frame). The muon's magnetic moment decreases relative to the electron by the same ratio, but the muon's magnetic field strength inside the loops increases by the same ratio.

The tau mass is $1776.8417 \times 10^{6} \frac{\mathrm{eV}}{\mathrm{c}^{2}}$, but unfortunately the magnetic dipole moment can't be measured (half-life is way too short) but if this model is correct, then the tau magnetic dipole moment should be:

$$
\begin{aligned}
& \text { Model Predicted Tau Magnetic Moment } \\
& =\left(4.74450 \times 10^{-18} \mathrm{Amp} \cdot \mathrm{m}^{2} \cdot \frac{\mathrm{eV}}{\mathrm{c}^{2}}\right) /\left(1776.8417 \times 10^{6} \frac{\mathrm{eV}}{\mathrm{c}^{2}}\right) \\
& =2.68839 \times 10^{-27} \mathrm{Amp} \cdot \mathrm{m}^{2}
\end{aligned}
$$

Note, a " $g$ " factor correction would increase this very slightly, just like it would for an electron or muon [8].

\section{Similar Models}

We have recently discovered earlier, similar models of the electron such as A. L. Parson’s Magneton Modelpublished in 1915 [14], as well as Mac Gregor's spherical model (which uses the same diameter as this model) originally published in 1992 and recently reprinted in 2013 [15]. Parson hypothesized a loop similar to our model, but it consisted of a loop of "continuous" charge, which has two problems i.e. without any discrete charge(s) within the loop, there can be no $\partial E / \partial t$ leading to a failure for both 1) a generation of magnetism as well as 2) a generation of virtual photons. Parson calculated a much larger loop radius of $1.5 \times 10^{-9} \mathrm{~cm}$ or roughly 38.8 times as large as we hypothesis. Mac Gregor on the other hand calculated almost exactly the same diameter as our model hypothesises, i.e. he calculated $d=7.723 \times 10^{-13} \mathrm{~m}$, and supported his conclusions with solid theory. One place where we differ is in the "form" of the electron. Mac Gregor hypothesizes a solid sphere, of the same diameter as our model, on which a charge rotates about its equator. Our model does not use such a sphere (or any other "scaffolding") because we can't see how it would form, what it would be made of, and because we believe that such a construct will ultimately prove to be unnecessary.

\section{Discussions}

1) Why does an electron and positron loop close on itself "only" at a diameter of $7.723 \times 10^{-13} \mathrm{~m}$ (derived from Equation (3))? This is the biggest question. A resonance type solution that involves two variables, which can only be solved simultaneously at the de Broglie basefrequency is one likely explanation. Perhaps Zitterbewegung type oscillations can only "resonate" at the electron's de Broglie base frequency due to interactions of a cycle of virtual photons/Bosons being created and then reabsorbed, or perhaps a Larmor type precessional frequency exists due to the interaction between the rotating charge and the electron's own magnetic field. Whatever the solution is, in addition to explaining the electron's mass, magnetic moment, and spin like this model does, it should also explain the mystery of why the smallest stable electric charge is exactly the same whether it is an electron, positron, proton or anti-proton.

2) The loop diameter of this model is much bigger than some theoretical estimates based on small discrepancies measured in the magnetic " $g$ " factor, and the loop diameter is also bigger than direct measurements of the electron's cross-section based on high energy collisions, but it is important to note that the loop diameter of this model is not the same as the size of the charged region. We have not addressed the size or shape of the charged region, but it would be this much smaller charged region that would affect transient measurements in high en- 
ergy collisions due to the fact that when the charged region is in a part of the loop moving away from the observer, it is doing so at the speed of light (can't be measured) and then when it is moving towards the observer, the gamma correction would make it look smaller to the observer (higher frequency observed, and shorter interaction time).

3) Can this model also help explain protons, neutrons and quarks? We think that when electron-positron pairs first formed from bosons after the Big Bang (only bosons could overlap enough to occupy the initial state), there must have been an equal number of electrons and positrons generated by high energy gamma ray pair-production. So where did the positrons go? We think the universe had to "tip" one way or the other, towards either predominantly regular matter or predominantly antimatter (a stable universe of both can't coexist), and in the process of tipping towards regular matter, the primordial positrons became bound up inside protons and neutrons. How? If you lay onto the palm of one hand 3 very strong spherical magnets of about $2 \mathrm{~cm}$ in diameter, and then drop 2 or 3 more magnets onto the others as shown in Figure 2 "Before" and "After" the magnets will tend to form themselves into toroid loops. This is because by forming a loop, the magnetic fields can self-reinforce into a more compact state. We propose that the higher magnetic fields within these toroids would allow the higher energy states like the Muonversion of the positron (and electron) to exist at rest (this would lead to a higher mass in a smaller space which fits with the fact that protons and neutrons have a smaller cross-section relative to electrons). A toroid composed of alternately electron and positron versions of the muon would want to self-annihilate due to the electrostatic forces, but if they are aligned magnetically, then the spin rotations are opposite to what would be needed for the formation of a photon. The increased magnetic field strength of the more compact muons might provide the stability to prevent electron and positron muons from recombining. Equations (14) and (15) can also be used to show that the magnetic attraction force of two positron muons would be greater than their electrostatic repelling force at that scale.

4) It is interesting to note that the ratio of the radius given by this model, to the Bohr radius [16] of the first electron orbital in a hydrogen atom, has a value of: $\frac{3.86159246 \times 10^{-13}}{5.2917721092 \times 10^{-11} \mathrm{~m}}=0.0072973522$, which is equal to $1 / 137.036$, i.e. this ratio is exactly the same as the fine structure constant. This connection to the fine structure constant is probably related to the electron's "amplitude for absorption of a photon".

5) This model looks classical, but we feel that it does not contradict quantum mechanical models. One argument against a "classical" loop model is that the quantum mechanical calculation of the gyromagnetic ratio is only $1 / 2$ the "classical" value. The error in this argument lies in the fact that it has been assumed that the mass of the electron would rotate at the same radius as the rotating charge. In our model the mass and gravity are due to the distortion of space time created by the electromagnetic energy densities, and since most of the electromagnetic energy lies well within the loop diameter, most of the mass would also lie inside the loop diameter. Another argument we have encountered contradicting the viability of this model is Bell's theorem. We feel that this argument is a similar straw-man comparison; i.e. if done properly, the misaligned spin of an electron would need a cosine correction when it encounters a detector, just the same as a quantum model would generate. A full discussion would more properly be reserved for a subsequent paper.
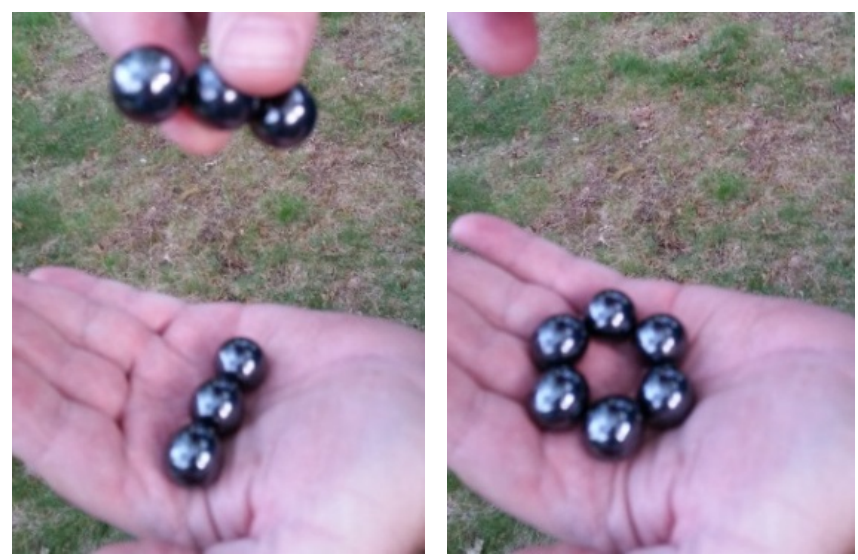

Figure 2. "Before” Magnets drop; “After” Magnets drop. 
6) At first glance this model doesn't account for linearly polarized photons. The general form of all photons however is elliptical [12], with either a right hand or a left hand rotation. Photons that are exactly circular or exactly linear are extreme cases of elliptical, and we believe simply hypothetical ideals. If for example one puts a circularly polarized filter after a linear filter, as shown in Figure 3, one can see that it does not become completely opaque and is only filtered to the same extent as the background, which indicates that the right hand circular filter is eliminating left hand photons from both the "linear" polarizer and the background roughly equally, and hence the "linear" polarized light is actually composed of a mix of right and left elliptical photons the same as the background. If a purely linear photon existed, it would be impossible for it to have a rotation, either right hand or left hand, and hence it would not be able to pass a left hand or a right hand circular filter.

A photon's ellipticity is also not constant; for example a (mostly) linear photon can easily be changed into a (mostly) circularly polarized photon by sending it through a quarter wave plate or other non-linear optics. The process of forming matter from photons is undoubtedly very nonlinear. It is possible that a certain degree of ellipticity may enhance the formation of matter, and future experiments testing this variable may give some interesting additional insight into the process of how matter is formed from photons.

Electrons and positrons by contrast are most likely not elliptical. We believe that they are made of the same electromagnetic ingredients as the $1.022 \mathrm{MeV}$ photon that they originated from, but the electron and positron loops resort to circular loops once the magnetic field lines can close back on themselves (photon magnetic field lines don't close on themselves). Once the magnetic field lines can close, the field lines trapped inside the loop will exert an outward force on the rotating charge, and since the distance around the loop is the Compton wavelength of a $511 \mathrm{KeV}$ photon, i.e. a fixed distance, the loop will be pushed out to a circular orbit which is the only orbit at which it would exactly match the magnetic moment of the electron/positron (for a given Compton wavelength perimeter, any other shape would generate too small of an area and hence too small of a magnetic moment).

7) There are many arguments in favour of this model: a) the interesting connection to the fine structure constant, b) a recent experiment that has shown that photons have a mass that is off-center [17], as our model predicts, c) the possibility of extending these concepts to model the muon and tau higher energy states of electrons and positrons, d) the fact that it shows how spin and charge are conserved through the process of pair production, and e) the model demonstrating the magnetic alignments and forces required to overcome the electrostatic attraction of an electron and positron pair at that scale. Arguments a) through e) are 5 good arguments in favour of this model, but the strongest argument for the validity of this model comes from $f$ ) the fact that it generates the de Broglie frequency of an electron to an accuracy of over 6 decimal places, while at the same time it correctly predicts the magnetic moment of the electron to over 6 decimal places. When one of these values matches reality to an accuracy of better than one in a million, it might be a happy coincidence, but when both figures match so exactly, the chances of coincidence are considered significantly diminished.

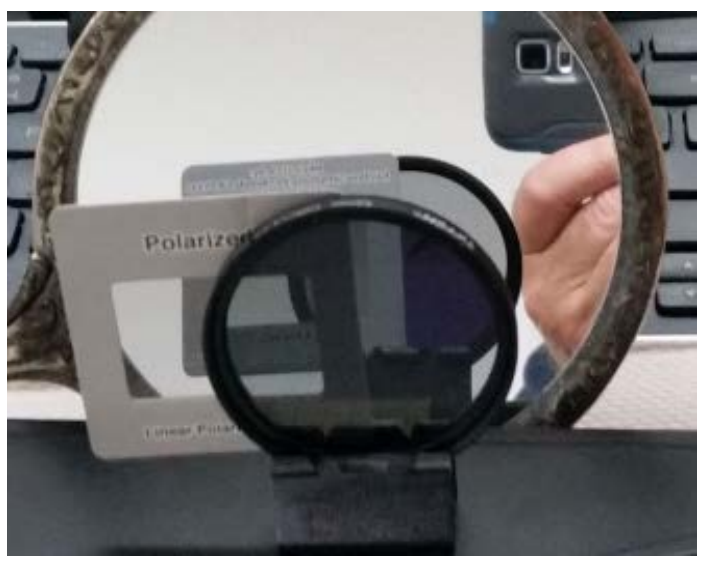

Figure 3. This image shows a circular polarized filter in front of a linear polarized filter in front of a mirror (mirror reverses circular polarization). The circular filter now blocks light through its own image, but it does not block light from a "linear" polarizer). 


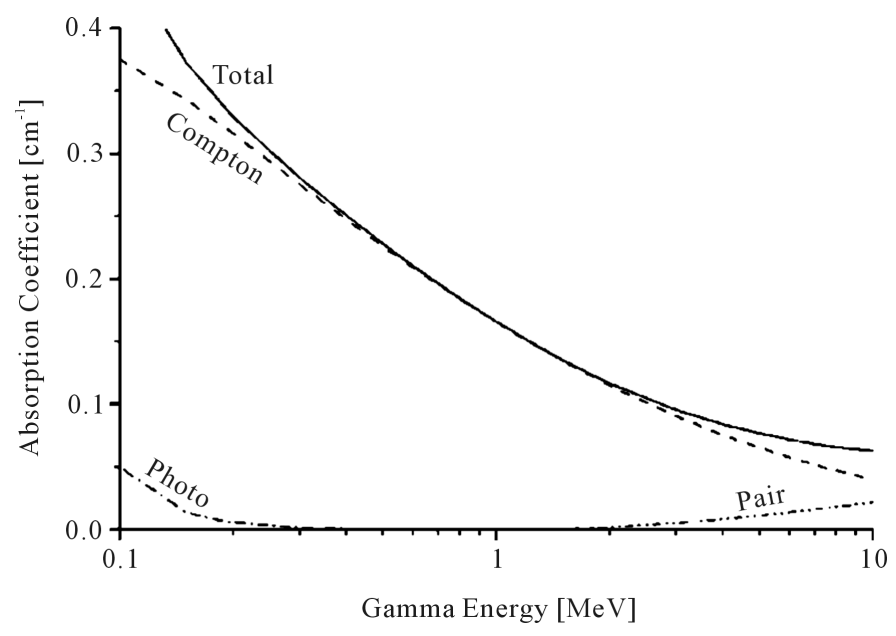

Graph 1. Electron-positron pair production in aluminum [2].

\section{Acknowledgements}

We would like to thank Walid Kyriakos PhD for helpful discussions.

\section{References}

[1] Maxwell, J.C. (1873) A Treatise on Electricity \& Magnetism Vol. II. Clarendon Press, London, 403.

[2] Paul, H. (2006) Gamma Absorption Coefficient for Aluminium. http://commons.wikimedia.org/wiki/File:Gamma_Abs_Al.png

[3] Classical Electron Radius (2014). http://en.wikipedia.org/wiki/Classical_electron_radius

[4] National Institute of Standards and Technology (2010) Elementary Charge. http://physics.nist.gov/cgi-bin/cuu/Value?e|search_for=electron+charge

[5] National Institute of Standards and Technology (2010) Bohr Magneton. http://physics.nist.gov/cgi-bin/cuu/Value?mub/search_for=elecmag_in

[6] National Institute of Standards \& Technology (2010) Electron Magnetic Moment Anomaly. http://physics.nist.gov/cgi-bin/cuu/Value?ae/search for=electron+anomaly

[7] Williamson, J.G. and van der Mark, M.B. (1997) Annales de la Fondation Louis de Broglie, 22, 133.

[8] Feynman, R.P. (1985) The Strange Theory of Light and Matter. Princeton University Press, Princeton, 115-118.

[9] Matter Wave (2014). http://en.wikipedia.org/wiki/Matter_wave

[10] De Broglie-Bohm Theory (2014). http://en.wikipedia.org/wiki/De_Broglie-Bohm_theory

[11] Jones, S. (2008) Chapter 16: The Quantum Ten. Oxford University Press, New York.

[12] Wikipedia (2014) Elliptical Polarization. http://en.wikipedia.org/wiki/Elliptical_polarization

[13] Wikipedia (2014) Maxwell's Equations. http://en.wikipedia.org/wiki/Maxwell's_equations

[14] Parson, A.L. (1915) Smithsonian Miscellaneous Collection, 65, 1-80.

[15] Mac Gregor, M.H. (2013) The Enigmatic Electron. 2nd Edition, El Mac Books, Santa Cruz.

[16] National Institute of Standards and Technology (2010) Bohr Radius. http://physics.nist.gov/cgi-bin/cuu/Value?bohrrada0|search_for=bohr+radius

[17] Agarwal, N.S. (2015) Journal of Modern Physics, 6, 627-633. http://file.scirp.org/Html/13-7502184_55832.htm 